\title{
Modelagem do Extintor da Caustificação de uma Indústria de Celulose por Regressão Linear Múltipla
}

\author{
Colombini, E. C. ${ }^{1 *}$; Pinheiro, O. S. ${ }^{2}$; Costa A. O. S. ${ }^{3}$; Costa Jr, E. F. ${ }^{3}$ \\ 1 Curso de Graduação em Engenharia Química, Universidade Federal do Espírito Santo, Alegre, ES, Brasil. \\ 2 Celulose Nipo-Brasileira S.A., Belo Oriente, MG, Brasil. \\ 3 Pós-Graduação em Engenharia Química e Graduação em Engenharia Química, Departamento de Engenharia Rural,
} Universidade Federal do Espírito Santo, ES, Brasil

* e-mail: cotta28@hotmail.com

\begin{abstract}
Resumo
O processo Kraft é um método de preparação de pasta celulósica cuja principal vantagem é a recuperação dos produtos químicos a ele associados. O sistema de caustificação faz parte do ciclo de recuperação química desse processo e, mesmo que esse sistema seja completado nos Caustificadores, grande parte da cal é convertida em $\mathrm{CaCO}_{3}$ no Extintor. O presente trabalho tem por objetivo gerar modelos empíricos com o auxílio da técnica estatística regressão linear múltipla a fim de tentar descrever de maneira mais eficaz a eficiência do Extintor. A partir de dados operacionais fornecidos por uma indústria de celulose $e$ utilizando-se também do software STATISTICA 7 que realizou os tratamentos estatísticos, foram gerados modelos estáticos e dinâmicos, mesmo sabendo que o processo de caustificação ocorre de maneira dinâmica. Nos modelos dinâmicos, a técnica utilizada foi a inserção da variável Eficiência de Caustificação como entrada atrasada, ou seja, adicionou-se valores de EC (lidos anteriormente) aos dados a serem previstos. Os resultados obtidos são apresentados ao final de cada modelo de regressão por meio dos coeficientes de determinação e também por gráficos para os dados de validação. Todos os modelos dinâmicos forneceram resultados superiores quando comparados aos estáticos, descrevendo de maneira satisfatória o Extintor.
\end{abstract}

Palavras chaves: modelagem computacional, extintor da caustificação, regressão linear múltipla

\begin{abstract}
The Kraft process is a method of preparing pulp whose main advantage is the recovery of chemicals associated therewith. The causticizing system is part of the chemical recovery cycle of this process, and even though this system is completed in Causticizers, most of the lime is converted into $\mathrm{CaCO}_{3}$ in the Slaker. This work aims to generate empirical models with the help of statistical technique multiple linear regression to try to describe more effectively the efficiency of the slaker. From operational data from a cellulose industry and also using up the STATISTICA 7 software that performed the statistical procedures were generated static and dynamic models, even though the causticizing process occurs dynamically. In dynamic models, the technique was used to insert the variable Causticizing Efficiency as late entry. In other words, added values of EC (previously read) in the data to being provided. The results obtained are presented at the end of each regression model by the determining coefficients and also by the graphics to the validation data. All dynamic models provide superior results when compared to static models, describing satisfactorily the slaker.
\end{abstract}

Keywords: computational modeling, causticizing slaker, multiple linear regression. 


\section{Introdução}

O setor de celulose e papel compreende uma importante fatia da economia do Brasil. A produção brasileira de celulose em 2013 totalizou mais de 15 milhões de toneladas, sofrendo um aumento de $8,2 \%$ em relação ao ano de 2012. Para os meses de Janeiro e Fevereiro de 2014, registrou-se cerca de $5 \%$ de aumento na produção em relação aos mesmos meses de 2013. Além disso, o saldo da balança comercial do setor de celulose e papel em 2013 foi de US\$ 5 milhões, 12,8\% maior que em 2012 [1].

Mais além, o Brasil foi o quarto maior produtor mundial de celulose em 2012, atrás apenas dos Estados Unidos, China e Canadá. Devido ao crescimento, desenvolvimento tecnológico e científico e qualificação da mão de obra do setor, houve um aumento na produtividade da madeira para celulose [1]. No período de 1980 a 2011 a produtividade do Eucalipto cresceu $83 \%$, enquanto a do Pinus aumentou $100 \%$. Além disso, o potencial de crescimento da produtividade brasileira é avaliado em $75 \%$ comparado com os números atuais [1].

Além dos dados estatísticos de mercado, é também preciso compreender a produção da celulose. O processo Kraft ou processo sulfato é um método de preparação de pasta celulósica desenvolvido por Dahl, na Alemanha, em 1879 [2]. O objetivo desse processo é dissolver a lignina - substância responsável pela ligação entre as fibras da madeira - com o mínimo de degradação dos carboidratos (celulose e hemicelulose).

A principal vantagem do processo Kraft é a recuperação dos produtos químicos a ele associados. O sistema de caustificação faz parte do ciclo de recuperação química desse processo [2]. Seu objetivo é a produção do licor branco, utilizado na dissolução da lignina, a partir de produtos químicos inorgânicos produzidos na caldeira de recuperação e no forno de cal [3]. Tal produção é alcançada pela adição de cal ao licor verde, por meio de reações de hidratação de cal e de caustificação - que ocorrem no Extintor e nos Caustificadores -, gerando como subproduto carbonato de cálcio [4]. Na etapa de hidratação (ou extinção), a cal ( $\mathrm{CaO})$ é dissolvida em água no Extintor para obter-se hidróxido de cálcio $\left(\mathrm{Ca}(\mathrm{OH})_{2}\right)$, ocorrendo também liberação de energia. Essa reação ocorre de maneira rápida e em temperaturas próximas a $1000^{\circ} \mathrm{C}$ [3]. Mesmo que o processo de caustificação seja completado nos Caustificadores, grande parte da cal já é convertida em carbonato de cálcio no Extintor. Isto é, a eficiência de caustificação nesse equipamento representa grande parte dos índices obtidos ao final do último caustificador.

Então, visto que esse ciclo de recuperação possui notável participação no processo Kraft e que o Extintor é parte imprescindível desse ciclo, esse trabalho tem por objetivo descrever de maneira mais eficaz a eficiência do Extintor do sistema de caustificação de uma indústria de celulose, gerando modelos empíricos - estáticos e dinâmicos - com o auxílio da técnica estatística regressão linear múltipla. Esses modelos são importantes para avaliar a influência das variáveis de entrada na eficiência de caustificação e, portanto, tornar o processo de recuperação mais eficaz.

\section{Materiais e Métodos}

Utilizando-se da regressão linear múltipla, foram construídos modelos empíricos, ou seja, que não são baseados em conceitos teóricos. Os materiais empregados para a geração desses modelos são os dados experimentais cedidos por uma indústria de celulose e o software STATISTICA 7 para realizar os tratamentos estatísticos.

A regressão múltipla é uma técnica estatística para investigar e modelar a relação entre variáveis e é amplamente usada em análise de dados, por exemplo [5]. De maneira geral, o objetivo dessa técnica é avaliar a relação de uma variável de interesse $Y$ (variável dependente ou variável resposta) em relação a $k$ variáveis $X_{j}$ (variáveis independentes ou covariáveis), tal que $j=1,2,3, \ldots, k$. Logo, a equação (1) é um possível modelo:

$$
y i=\beta_{0}+\beta_{1} \cdot x_{i 1}+\beta_{2} \cdot x_{i 2}+\cdots+\beta_{k} \cdot x_{i k}+\varepsilon_{i}
$$

Em que:

- $\quad i=1, \ldots, \mathrm{n}$;

- $\quad n$ é o número de pontos experimentais;

- $\quad y_{i}$ é a observação da variável dependente para o i-ésimo ponto experimental;

- $\quad X_{i}=\left(x_{i 1}, X_{i 2}, \ldots, x_{i k}\right)^{\prime}$ é um vetor de observações das variáveis independentes para o i-ésimo ponto experimental;

- $\quad \beta=\left(\beta_{0}, \beta_{1}, \beta_{2}, \ldots, \beta_{k}\right)^{\prime}$ é um vetor de coeficientes de regressão (parâmetros);

- $\quad \varepsilon_{i}$ é um componente de erro aleatório. Assumese que esses erros são independentes e seguem distribuição normal com média zero e variância desconhecida $\sigma^{2}$.

Além disso, existem observações acerca dessa técnica: i) Para estimar os coeficientes de regressão em (1), pode utilizar-se o método dos mínimos quadrados (MMQ); ii) A equação (1) é chamada de Regressão Linear Múltipla pois envolve mais de um coeficiente de regressão; iii) $O$ termo "Linear" indica que o modelo é linear em relação aos parâmetros $\beta=\left(\beta_{0}, \beta_{1}, \beta_{2}, \ldots, \beta_{k}\right)$ e não porque $y$ é uma função linear dos $x$ 's [5].

Um parâmetro usado para julgar a adequação de um modelo de regressão é o Coeficiente de Determinação $\left(R^{2}\right)$, cujo intervalo de compreensão é $0 \leq R^{2} \leq 1$. 
Casualmente, quanto mais próximo de 1 for $\mathrm{R}^{2}$, melhor é considerado o modelo, ou seja, as variáveis independentes possuem uma relação bastante linear com a variável dependente. Entretanto, $R^{2}$ não é um medidor exato de adequação do modelo e, portanto, em algumas situações específicas, um valor grande do coeficiente pode não garantir que o modelo de regressão fornecerá previsões precisas das observações [6].

As variáveis utilizadas na construção dos modelos estão apresentadas na Tabela 1. Para os modelos dinâmicos, foram empregadas $\mathrm{EC}(-1)$ e TS(-1), ambas como variáveis de entrada.

\begin{tabular}{|c|c|c|}
\hline Dados gerais & $\begin{array}{c}\text { Análise do Licor } \\
\text { Verde }\end{array}$ & $\begin{array}{l}\text { Dados do } \\
\text { Extintor }\end{array}$ \\
\hline $\begin{array}{l}\text { Vazão do Licor } \\
\text { (VL) }\left(\mathrm{m}^{3} / \mathrm{h}\right), \\
\text { Dosagem de } \\
\text { Cal (DC) } \\
\left(\mathrm{kg} / \mathrm{m}^{3}\right), \mathrm{Cal} \\
\text { Útil }(\mathrm{CU}) \\
(\% \mathrm{CaO} \text { na } \\
\text { Cal). }\end{array}$ & $\begin{array}{c}\text { Álcali efetivo (AE), } \\
\text { Álcali Ativo (AA), } \\
\text { Álcali total titulável } \\
\text { (ATT), Eficiência } \\
\text { de Caustificação } \\
\text { (EC1), Sulfidez } \\
\text { (S), } \mathrm{Na}_{2} \mathrm{CO}_{3}, \\
\mathrm{NaOH}, \mathrm{Na}_{2} \mathrm{~S} \text {. }\end{array}$ & $\begin{array}{l}\text { Temperatura de } \\
\text { entrada no } \\
\text { extintor (TE), } \\
\text { Temperatura de } \\
\text { saída do } \\
\text { extintor (TS), } \\
\text { Eficiência de } \\
\text { Caustificação } \\
\text { (EC). }\end{array}$ \\
\hline
\end{tabular}

As variáveis $A E, A A, A T T, E C 1$ e $S$ são combinações das concentrações de $\mathrm{Na}_{2} \mathrm{CO}_{3}, \mathrm{NaOH}$ e $\mathrm{Na}_{2} \mathrm{~S}$. A variável dependente foi a Eficiência de Caustificação do Extintor (EC)

\section{Resultados e Discussão}

Foram construídos 5 modelos. Nos três primeiros não se utilizou entradas atrasadas, mesmo sabendo que o processo de caustificação ocorre de forma dinâmica. Ao elaborar tais modelos estáticos, assume-se que as entradas não variam no tempo, ou seja, o processo está em estado estacionário.

$\mathrm{Na}$ construção do Modelo 1, foram utilizadas todas as variáveis de entrada, exceto a Temperatura de Saída (TS), uma vez que sua medição é feita ao final do processo. A variável de saída foi EC. Os resultados obtidos foram: $R=0,5068, \quad R^{2}=0,2569 \quad$ e $R^{2}$ ajustado=0,2555. A partir desses valores pode-se concluir que o Modelo 1 se mostrou pouco eficiente.

Fez-se então uma análise mais detalhada dos dados e verificou-se a existência de valores muito pequenos (e até mesmo negativos) da variável Vazão de Licor (VL). Admitindo erro nas leituras ou também que não houve leitura nesses casos, tais dados foram excluídos e todos os modelos seguintes não contam com os mesmos. A partir desse corte, gerou-se o Modelo 2 com as mesmas variáveis empregadas no primeiro. Os resultados foram: $R=0,5204, \quad R^{2}=0,2708$ e $R^{2}$ ajustado=0,2692, representando uma pequena melhora em relação ao Modelo 1, mas pouco significativa ainda.

Antes de se elaborar o terceiro modelo, observou-se significantes repetições nos valores de EC, caracterizando, assim, a existência de dados não lidos. Esses dados foram então excluídos dos seguintes modelos. Eliminou-se também valores com mais de 10 intervalos de repetição, ou seja, dados que eram escritos após uma sequência de 10 ou mais repetições seguidas de outros valores. O conjunto final de dados a ser usado nos seguintes testes é composto por 1334 dados, sendo $90 \%$ destinados às regressões e $10 \%$ para as validações.

Após toda essa análise, foi gerado o Modelo 3 com as mesmas variáveis empregadas no primeiro e no segundo caso. Os resultados: $R=0,5038, R^{2}=0,2538$, $R^{2}$ ajustado=0,2505. É possível notar que os valores do coeficiente de determinação foram menores nesse caso em relação ao Modelo 2. Isso pode ter ocorrido devido à diminuição brusca dos dados do Modelo 2 para 0 terceiro caso. Na Figura 1, pode-se ver o gráfico de validação do Modelo 3.

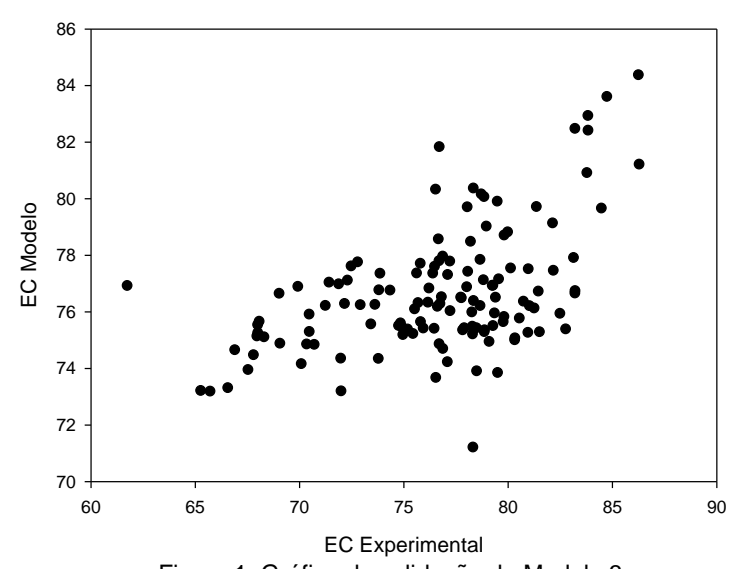

Figura 1: Gráfico de validação do Modelo 3.

Vale lembrar que todos os modelos gerados até aqui foram estáticos. Entretanto, como já mencionado que o processo de extinção é dinâmico, para o quarto modelo inseriu-se a variável EC(-1) como entrada. Além dela, inseriu-se as 12 variáveis originais e também EC (com os cortes descritos anteriormente). Os resultados obtidos foram: $\quad R=0,9185, \quad R^{2}=0,8437$, $R^{2}$ ajustado=0,8429. Analisando os coeficientes de determinação, é possível observar a melhora frente os resultados obtidos nos modelos estáticos anteriores. $\mathrm{A}$ Figura 2 apresenta o gráfico de validação do Modelo 4. 


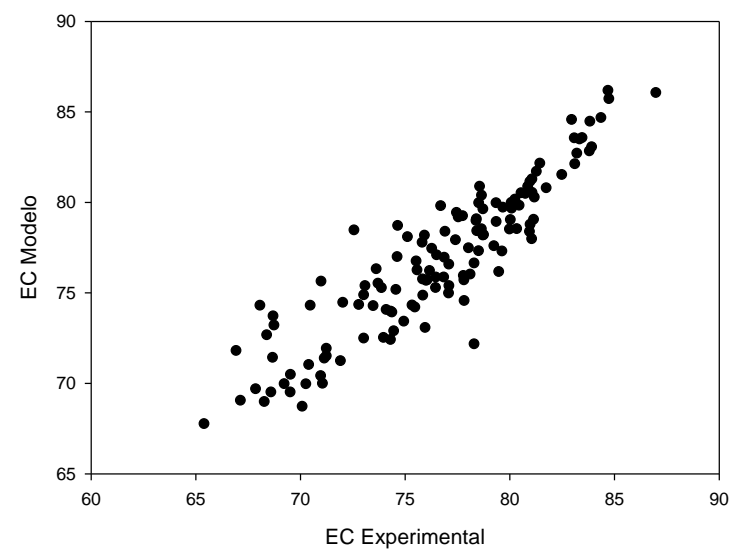

Figura 2: Gráfico de validação do Modelo 4.

No Modelo 5, além das variáveis utilizadas anteriormente no Modelo 4, empregou-se também como entrada a Temperatura de Saída do Extintor atrasada TS(-1), uma vez que os valores empregados para o modelo já teriam sido lidos e não estariam mais ao final de cada intervalo do processo. Os resultados do Modelo 5: $R=0,9292, R^{2}=0,8633, R^{2}$ ajustado=0,8623. A Figura 3 apresenta o gráfico de validação do Modelo 5 .

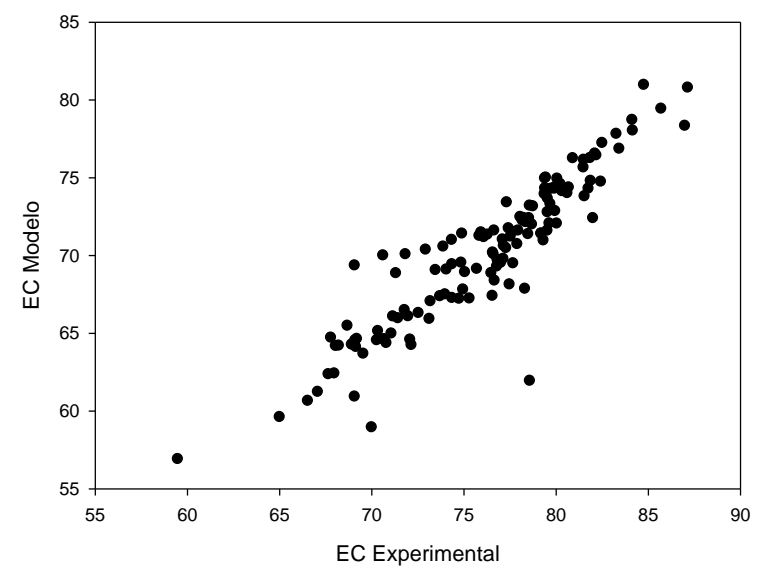

Figura 3: Gráfico de validação do Modelo 5.

É possível perceber, a partir dos coeficientes de determinação e gráficos de validação, que o Modelo 5 consegue melhor descrever o processo em análise. A representação matemática do quinto modelo está apresentada na Equação 2.

$$
\begin{aligned}
& E C=-18,0028+0,8274 \cdot E C(-1)+0,6301 \\
& T S(-1)-0,1785 \cdot \mathrm{Na}_{2} \mathrm{CO}_{3}-0,0085 \cdot V L- \\
& 0,4049 \cdot S-0,1444 \cdot \mathrm{TE}+0,0390 \cdot \mathrm{CU}
\end{aligned}
$$

aos modelos estáticos. Isto é, a variável Eficiência de Caustificação do Extintor (EC) e, portanto, o Extintor, foram descritos de maneira satisfatória por modelos dinâmicos. Conclui-se também que o melhor modelo obtido, embora tenha uma equação relativamente simples, descreve o sistema de maneira satisfatória com $\mathrm{R}^{2}$ de 0,8633 .

\section{Agradecimentos}

Os autores agradecem ao CNPq pela bolsa IC e pela bolsa de produtividade DTI e à FAPES pela bolsa pesquisador capixaba.

\section{Referências}

[1] ASSOCIAÇÃO BRASILEIRA DE CELULOSE E
PAPEL. PAPEL. Disponível
<http://bracelpa.org.br/bra2/index.php>. Acesso em: 11 Abr. 2015.

[2] ASSUMPÇÃO, R. M. V.; PINHO, M. R. R.; CAHEN, R.; PHILIPP, P. Polpação química. Celulose e papel. Tecnologia de fabricação de pasta celulósica, Volume 1, 2ª ed., São Paulo: IPT: 1988.

[3] FIGUEIRÊDO, L. S. Modelagem matemática do estado estacionário de um real sistema de caustificação em uma fábrica de celulose. $71 \mathrm{f}$. Dissertação (Mestrado em Engenharia Industrial), Programa de Pós-Graduação em Engenharia, Centro Universitário do Leste de Minas Gerais, Coronel Fabriciano, 2009.

[4] CAMPOS, S. G. S. Modelagem matemática e análise do coeficiente global de transferência de calor para o processo de concentração do licor negro de eucalipto em sistema de evaporadores de múltiplo efeito. $110 \mathrm{f}$. Dissertação (Mestrado em Engenharia Industrial), Programa de Pós-Graduação em Engenharia, Centro Universitário do Leste de Minas Gerais, Coronel Fabriciano, 2009.

[5] BARROS, E. A. C. et al. Revista Colombiana de Estadística, v. 31, p. 111-129, 2008.

[6] MONTGOMERY, D.C.; RUNGER, G.C. Applied Statistics and Probability for Engineers. Nova lorque: John Wileyand Sons: 2003.

\section{Conclusões}

Em todas as análises realizadas, os modelos não estáticos, em que se inseriu as variáveis com atrasos, forneceram resultados superiores quando comparados 